\title{
Lack of cold pressor test-induced effect on visual-evoked potentials in migraine
}

\author{
Gianluca Coppola $\cdot$ Antonio Currà $\cdot$ Mariano Serrao • \\ Cherubino Di Lorenzo - Manuela Gorini - Elisa Porretta • \\ Alessia Alibardi · Vincenzo Parisi · Francesco Pierelli
}

Received: 21 October 2009/Accepted: 19 November 2009/Published online: 10 December 2009

(C) Springer-Verlag 2009

\begin{abstract}
In patients with migraine, the various sensory stimulation modalities, including visual stimuli, invariably fail to elicit the normal response habituation. Whether this lack of habituation depends on abnormal activity in the sub-cortical structures responsible for processing incoming information as well as nociception and antinociception or on abnormal cortical excitability per se remains debateable. To find out whether inducing tonic pain in the hand by cold pressure test (CPT) alters the lack of visual-evoked potential (VEP) habituation in migraineurs without aura studied between attacks we recorded VEPs in 19 healthy subjects and in 12 migraine patients during four experimental conditions: baseline; no-pain (hand held in warm water, $25^{\circ} \mathrm{C}$ ); pain (hand held in cold water, $2-4^{\circ} \mathrm{C}$ ); and after-effects. We measured P100 amplitudes from six blocks of 100 sweeps, and assessed habituation from
\end{abstract}

G. Coppola $(\bowtie)$ V. Parisi

Department of Neurophysiology of Vision and

Neurophthalmology, G. B. Bietti Eye Foundation,

IRCCS, Via Livenza 3, 00198 Rome, Italy

e-mail: gianluca.coppola@gmail.com;

gianluca.coppola@uniroma1.it

A. Currà $\cdot$ M. Gorini · A. Alibardi

Department of Neurological Sciences, Ospedale A. Fiorini-Polo

Pontino, "Sapienza" University, Rome, Italy

M. Serrao · C. Di Lorenzo - E. Porretta - F. Pierelli

University Centre for Adaptive Disorders and Headache

(UCADH), "Sapienza" University of Rome, Polo Pontino,

ICOT, Latina, Italy

M. Serrao · F. Pierelli

IRCCS Neuromed, Pozzilli (IS), Italy

V. Parisi

University of Rome "Campus Biomedico", Rome, Italy amplitude changes between the six sequential blocks. In healthy subjects, the CPT decreased block 1 VEP amplitude and abolished the normal VEP habituation (amplitude decrease to repeated stimulation) in patients with migraine studied between attacks; it left block 1 VEP amplitude and abnormal VEP habituation unchanged. These findings suggest that the interictal cortical dysfunction induced by migraine prevents the cortical changes induced by tonic painful stimulation both during pain and after pain ends. Because such cortical changes presumably reflect plasticity mechanisms in the stimulated cortex, our study suggests altered plasticity of sensory cortices in migraine. Whether this abnormality reflects abnormal functional activity in the subcortical structures subserving tonic pain activation remains conjectural.

Keywords Migraine - Cold pressor test .

Visual-evoked potentials · Habituation - Brainstem .

Thalamo-cortical activity

\section{Introduction}

Growing experimental evidence shows that in episodic migraineurs without aura, repetitive stimulation delivered with various sensory modalities during the pain-free interval elicits abnormal cortical responses characterized by deficient habituation or adaptation [1,2]. Habituation is a ubiquitous phenomenon observed in simple and complex neuronal circuits, commonly defined as "a response decrement as a result of repeated stimulation" [3]. Although habituation takes place at various nervous system levels including the cerebral cortex, the mechanisms responsible for decreasing responses to sensory stimuli remain poorly understood [4]. This uncertainty helps explain why 
abnormal habituation in migraine still lacks a definitive consensus interpretation. Current ideas attribute this neurophysiological abnormality to cortical hyper-excitability probably arising from deficient intracortical inhibition $[5,6]$, or to low sensory cortical pre-activation levels ultimately due to abnormal functioning of monoaminergic projections from the brainstem $[1,2]$.

In migraine, the lack of habituation manifests when patients are pain-free (i.e. during the interictal or pre-ictal phase), culminates just before the onset of an attack [7-9], and disappears during the attack [7,9-11], giving way to cortical responses to repetitive stimulation that transiently normalize. In migraineurs pain therefore helps to normalize cortical response amplitudes to repeated stimulation.

The current standard method for assessing excitability in the occipital cortex entails recording visual-evoked potentials (VEPs), electrical cortical responses to monocular visual stimuli. In a study aimed to examine the effect of pain on VEPs we recently showed that inducing pain in healthy volunteers abolished habituation even in cortical areas not involved in pain processing [12]. When the healthy subjects we studied underwent to the cold pressor test (CPT) - a long used and validated in vivo model for studying nociception and antinociception [13-15]—we found that the tonic pain induced in the hand reduced VEP amplitudes and abolished normal VEP habituation in the occipital cortex, a phenomenon that persisted after the painful stimulation ended. We attributed these changes in visual cortex excitability to pain-induced activation of brainstem monoaminergic nuclei that in turn modulates thalamic and thalamo-cortical neuronal activity, thus enacting a complex behavioral response aimed to adopt defensive strategies against pain.

Evidence that pain impairs habituation of cortical responses to repeated stimulation in healthy subjects but promotes habituation in migraineurs suggests that the structures subserving tonic pain act differently in patients with migraine and healthy subjects. Previous studies have nevertheless reported discrepant results [16-18] probably owing to the non-homogeneous techniques used for inducing pain (CPT or capsaicin application), and the differing neurophysiological variables studied (trigeminocervico-spinal, noxious flexor or blink reflexes). Most important, none of these studies explored the cortical circuits in which pain is known to change how the responses habituate to repetitive stimulation [12].

More precise data are therefore needed to show how pain modulate cortical activity in migraineurs and healthy subjects, and especially to see whether activating the structures subserving tonic pain alters the disease-related changes in cortex excitability in migraineurs without aura. Studying cortical responses during tonic pain in migraineurs offers the unique opportunity to investigate visual cortex responsivity under conditions when baseline habituation is absent [1] and baseline activity in brainstem serotonergic nuclei reduced [19].

We designed this study to find out whether inducing tonic pain in the hand by CPT alters the lack of VEP habituation in migraineurs without aura studied between attacks. To do so, we compared VEP-CPT-induced changes in migraineurs without aura studied interictally and healthy subjects.

\section{Methods}

\section{Subjects}

We enrolled a group of 14 migraine patients without aura (MO, ICHD-II code 1.1) who underwent VEP recordings during the interictal period, i.e. attack-free for at least 3 days before and after the recording sessions, and a group of 18 healthy subjects recruited among students attending the medical school and healthcare professionals. Inclusion criteria were absence of any overt medical condition, and in particular, any personal or family history of neurological (including migraine, for the healthy subjects) or psychiatric illness. Because neurophysiological investigations show that pain modulation changes during the menstrual cycle [20], we included only female participants in the follicular phase.

All participants received a complete description of the study and granted informed consent, and the project was approved by the Ethics Committee of the "Sapienza" University of Rome, Polo Pontino. Participants taking regular medications were excluded, as well as subjects who failed to reach a best corrected visual acuity of $>8 / 10$.

\section{Visual-evoked potentials}

Subjects sat in a semi-dark, acoustically isolated room in front of the display surrounded by a uniform luminance field of $5 \mathrm{~cd} / \mathrm{m}^{2}$. To obtain a constant pupil diameter, before VEP recording, each subject adapted to the ambient room light for $10 \mathrm{~min}$. VEPs were recorded to monocular stimulation with the contralateral eye occluded. Visual stimuli consisted of full-field checkerboard patterns (contrast $80 \%$, mean luminance $250 \mathrm{~cd} / \mathrm{m}^{2}$ ) generated on a TV monitor and reversed in contrast at a rate of 3.1/s. At the viewing distance of $114 \mathrm{~cm}$, the single check edges subtended a visual angle of $15^{\prime}$. Subjects were instructed to fixate a red dot in the middle of the screen with the contralateral eye covered by a patch to maintain stable fixation. VEPs were recorded from the scalp through silver cup electrodes positioned at $\mathrm{Oz}$ (active electrode) and at $\mathrm{Fz}$ (reference electrode, 10/20 system); and a ground electrode 
was placed on the right forearm. Signals were amplified by Digitimer $^{\mathrm{TM}}$ D360 pre-amplifiers (band-pass 0.05$2,000 \mathrm{~Hz}$, gain 1,000$)$ and recorded by a $\mathrm{CED}^{\mathrm{TM}}$ power 1401 device (Cambridge Electronic Design Ltd, Cambridge, UK). A total 600 consecutive sweeps each lasting $200 \mathrm{~ms}$ were collected and sampled at 4,000 Hz.

Cortical responses were partitioned in six sequential blocks of 100, consisting of at least 95 artifact-free sweeps. Responses in each block were averaged off-line ("block averages") using the Signal ${ }^{\mathrm{TM}}$ software package version 3.10 (CED Ltd).

VEP components were identified according to their latencies from the stimulus: N1 was defined as the most negative peak between 60 and $90 \mathrm{~ms}, \mathrm{P} 1$ as the most positive peak following N1 between 80 and $120 \mathrm{~ms}$, and N2 as the most negative peak following P1 at between 125 and $150 \mathrm{~ms}$ (Fig. 1). We measured the peak-to-peak amplitude of the N1-P1 complex. Habituation was defined both as the change in amplitude of N1-P1 recorded during the six blocks and the slope of the linear regression line for the six blocks.

\section{Cold pressor test}

We induced tonic pain using a validated cold pressor test (CPT) $[14,15,21]$. The subjects were required to dip their right hand, to a depth of $5 \mathrm{~cm}$ above the wrist, in a thermoregulated water bath for $4 \mathrm{~min}$. The water temperature was maintained at $3-4^{\circ} \mathrm{C}$.

Procedure

VEP habituation was evaluated in separate sessions under four experimental conditions: baseline, before the CPT; nopain condition (VEPs recorded while subjects dipped their hand in the water at $25^{\circ} \mathrm{C}$ ); pain condition (VEPs recorded 1 min after the CPT started); and after-effects condition (VEPs recorded 5 min after the CPT ended). Pain and nopain conditions were randomly assigned after the baseline session. To avoid possible skin receptor sensitization, after the hand was removed from the water it was dried, and subjects rested more than 20 min between pain and no-pain sessions. Because the no-pain condition probably does not sensitize skin receptors, after the hand was removed from the water subjects rested about 10 min between no-pain and pain sessions (Fig. 2). All recordings were collected in the morning (between 09.00 and 11.00 a.m.) by the same experimenter.

Subjects rated the subjective intensity of the painful sensation on an 11-point numerical scale (11P NS) scale, graded from 0 no pain to 10 unbearable pain.
Fig. 1 Representative recordings of visual-evoked potential (VEP) habituation at baseline (left) and during the cold pressor test (right) in a healthy subject (HS) and a migraine patient without aura (MO)
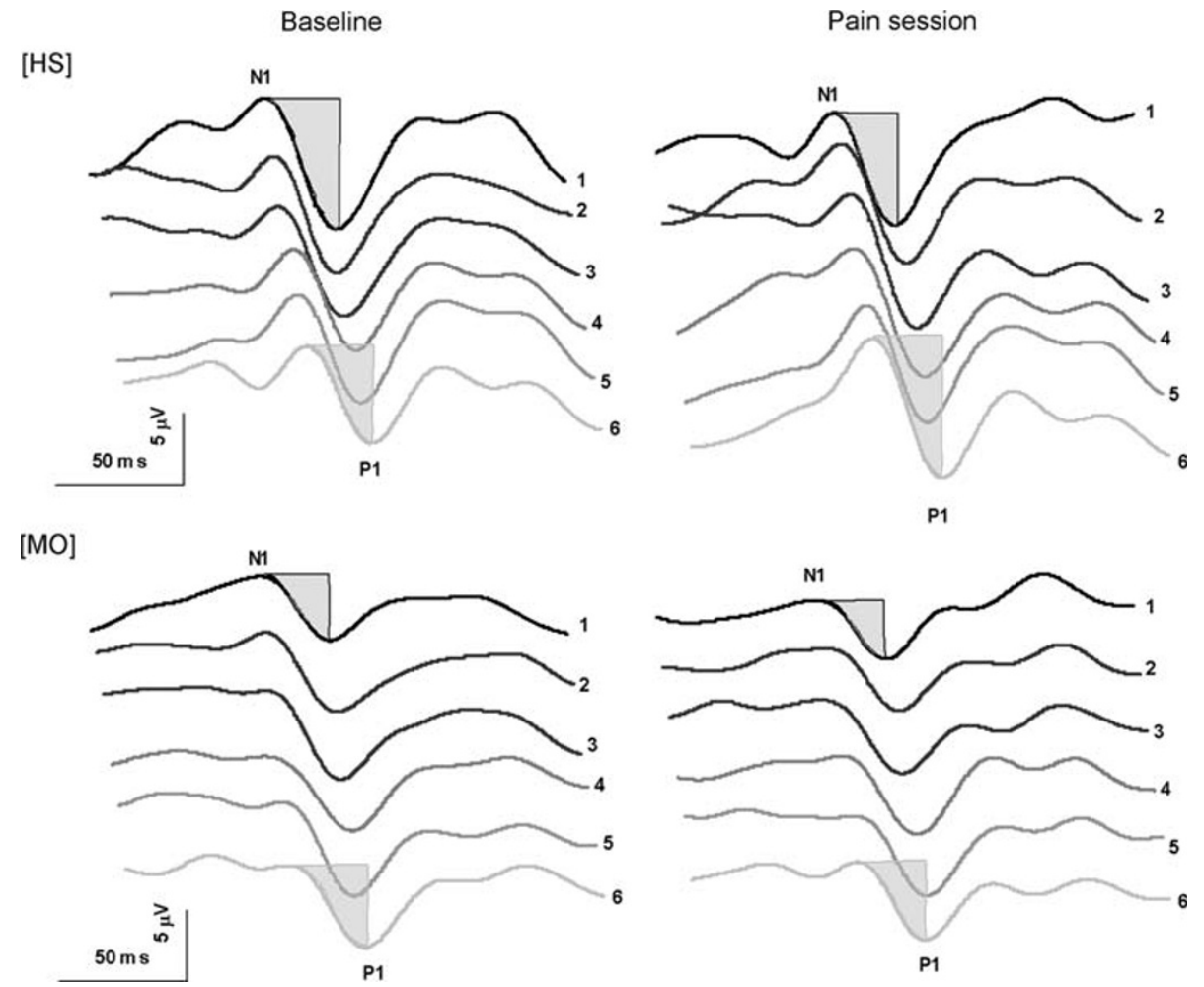

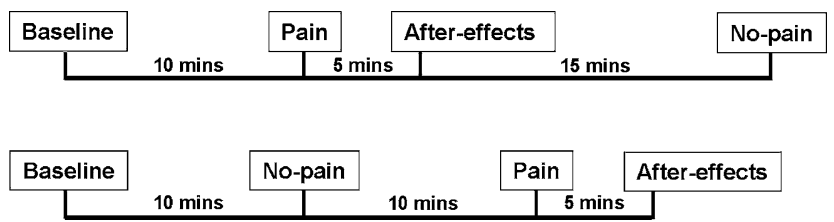

Fig. 2 Flow chart of the study procedure

\section{Statistical analysis}

We used the Statistical Package for the Social Sciences (SPSS) for Windows, version 15.0 for all analyses. We performed multivariate analysis of variance taking as a within-subject factor "block" and as between-subject factors "Group" (HS, MO) and "condition" (baseline, pain, no-pain and after-effect). A regression analysis was used to disclose linear trends in VEP amplitude across blocks in each condition and group (slope). Paired-sample $t$ test was used to compare block 1 VEP amplitude at baseline and during pain condition in both groups. The Fisher least significant difference (LSD) test was used for post hoc analysis. Mann-Whitney $U$ test was used to compare 11 PNS scores between groups. Pearson's correlation coefficient was used to test correlations between VEP habituation and subjective pain measures (11 PNS scores). $P$ values less than 0.05 were considered to indicate statistical significance.

\section{Results}

Of the 32 participants initially enrolled, two patients and two healthy subjects who found the pain intolerable withdrew before the painful recording session ended and their data were excluded from the analysis. Complete VEP recordings were obtained for 12 migraineurs and 19 healthy subjects (Table 1).

In healthy subjects and patients with $\mathrm{MO}(7.7 \pm 2.0$ and $8.1 \pm 1.4 ; Z=-0.99 ; P=0.322) 11$ PNS scores for subjective pain were similar.

ANOVA testing VEP amplitude block averages disclosed a main effect for factor block $\left(F_{(5,575)}=3.59\right.$,

Table 1 Clinical and demographic data of healthy volunteers (HV) and migraine without aura patients (MO)

\begin{tabular}{lll}
\hline & HV $(n=19)$ & MO $(n=12)$ \\
\hline Women $(n)$ & 10 & 8 \\
Age (years) & $26.2 \pm 3.5$ & $28.1 \pm 5.8$ \\
Duration of history of migraine (years) & & $14.0 \pm 5.1$ \\
Attacks frequency/month $(n)$ & & $1.9 \pm 2.1$ \\
Attacks duration (h) & & $21.4 \pm 21.1$ \\
\hline
\end{tabular}

Data expressed as mean $\pm \mathrm{SD}$
$P=0.003$ ), and a significant two-way interaction of group by block $\left(F_{(5,575)}=4.31, P<0.001\right)$. Linear regression analysis of VEP amplitudes recorded in all blocks differed between sessions in healthy subjects $\left(F_{(3,72)}=3.25\right.$, $P=0.02)$ but not in patients $\left(F_{(3,43)}=0.61, P=0.61\right)$. Post hoc analysis showed that during baseline and no-pain conditions in healthy subjects the linear trend in VEP amplitudes decreased from block 1 to block $6(-0.28$ during baseline, -0.18 during no-pain), whereas in patients it increased $(+0.008$ during baseline, +0.19 during nopain; Fig. 3). Conversely, during the pain condition and after-effects, the linear trend in VEP amplitudes remained unchanged or almost unchanged (pain condition healthy subjects 0.00 , MO +0.09 ; after effect healthy subjects $-0.11, \mathrm{MO}+0.009)$ in both groups.

Paired $t$ test showed that the baseline block 1 VEP amplitude decreased significantly during the pain condition in healthy subjects $\left(t_{(1,18)}=3.53, P=0.002\right)$ but not in patients $\left(t_{(1,18)}=2.02, P=0.08\right)$.

Pearson's test disclosed no correlation between the 11 PNS scores and slopes of VEP amplitude linear trends in both groups (all, $P>0.05$ ).

\section{Discussion}

Our results in this study show that activating the structures subserving tonic pain leaves the disease-related changes in visual cortex excitability in migraineurs without aura unaltered. Whereas in healthy subjects, the CPT decreased block 1 VEP amplitude and abolished the normal VEP habituation (amplitude decrease to repeated stimulation), in patients with migraine studied between attacks, it left block 1 VEP amplitude and abnormal VEP habituation unchanged.

The findings of the present study in healthy subjects confirm our previous observation that tonic pain modulates the cortical response to repeated visual stimuli and does so by inducing an early depression and a late facilitation (loss of habituation) of cortical response amplitude that persists for several minutes after pain ends [12]. This interpretation finds support in electroencephalographic (EEG) and eventrelated desynchronization (ERD) studies of cortical function showing that tonic pain induces marked changes in posterior areas [22-25]. It also harmonizes nicely with the Thompson and Spencer [3] dual process theory that two opposing processes, i.e. depression and facilitation, predominate one after the other to determine the final behavioral outcome after stimulus repetition. At synaptic level, the stimulusresponse pathway (i.e. visual pathway) interacts with an external "state" system represented by various "tonic" nonspecific and motivational circuits (i.e. ascending neural systems including brainstem-thalamic circuits), critically 
Fig. 3 Visual-evoked potential N1-P1 block amplitudes during the four experimental conditions in healthy subjects (HS) and patients with migraine without aura (MO). The dotted line represents the slope of the linear regression lines

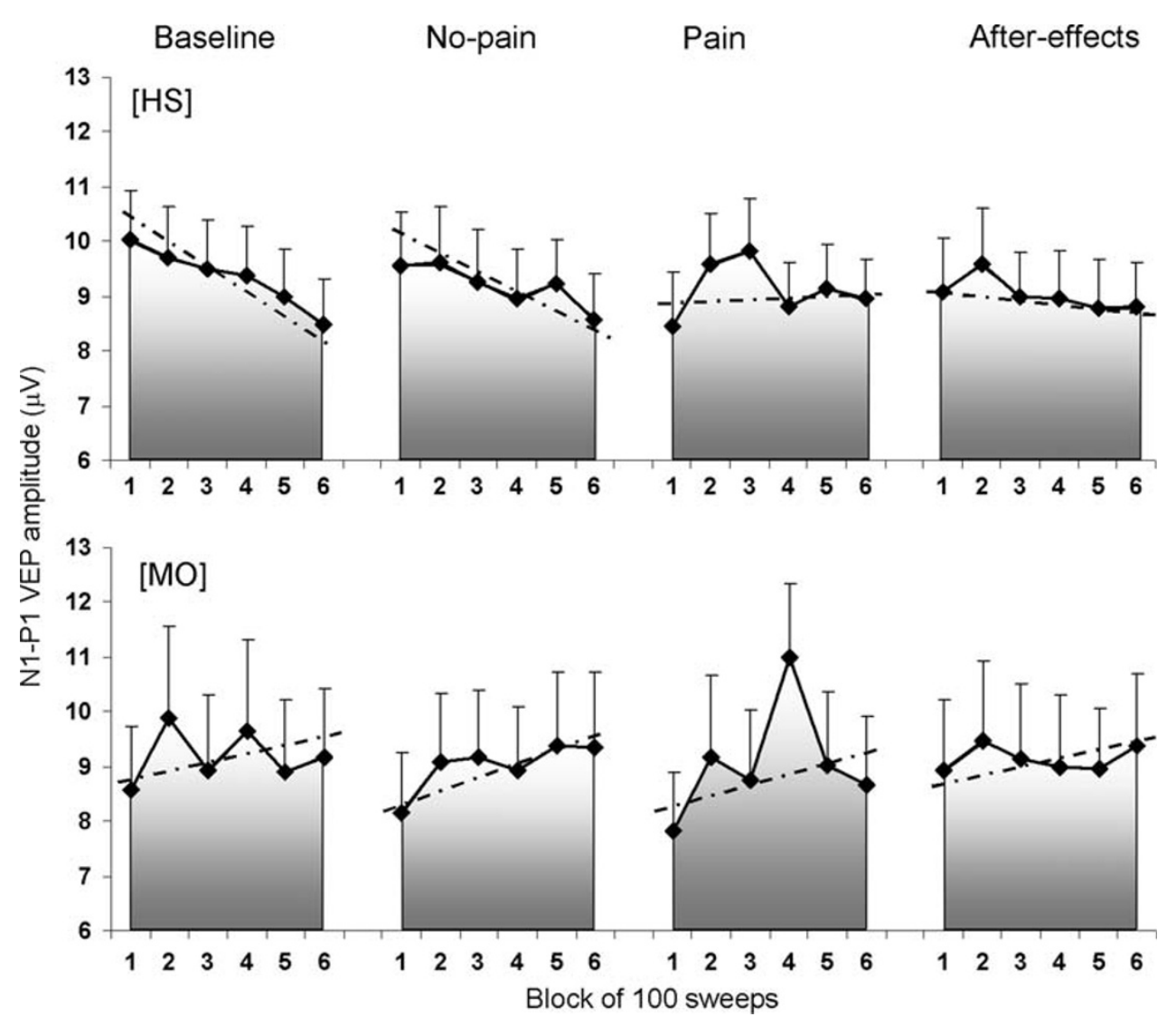

involved in the central processing of arousal, control of the signal-to-noise ratio generated by sensory stimuli at cortical and thalamic level, and endogenous antinociception [26, 27]. Changing cortical excitability during and after pain is part of a complex behavioral response aimed to adopt defensive strategies against pain. Inhibition of the early responses is probably driven by attentional processes to pain [28], whereas lack of habituation reflects the disruption of protective mechanisms whereby neuronal stress and toxic metabolites (i.e. lactate and protons) are prevented from accumulating in the sensory cortices after repeated stimulation and intervening pain [29].

Hence in healthy subjects CPT abolishes normal VEP habituation and does so likely by activating brainstem aminergic nuclei. Interestingly, evidence has accumulated showing that migraine is a disorder with low CNS serotonin transmission [19], and the latter was claimed as a possible explanation for the reduced VEP habituation [1, 2]. At first glance, our findings in migraineurs during the pain condition fit in well with a normal visual cortex response to pain, manifesting with a lack of habituation to repeated stimulation similar to that observed in healthy volunteers. But if the migraineurs cortex behaves "normally" during pain insofar as it lacks habituation, it differs from normal because it lacks the normal block 1 VEP amplitude reduction. This is a substantial difference because the neural mechanisms thought to sub-serve the early response depression trigger the processes causing late responses to recover or augment (i.e. lack of habituation). Our finding that tonic pain in the hand fails to reduce block 1 VEP amplitude in migraineurs therefore suggests that migraine patients lack the early cortical changes manifesting as a phasic decrease in alpha activity and seen in EEG and ERD studies in healthy subjects during the first minute after immersing the hand in cold water $[22,23]$. Consistently, during sustained photic stimulation migraineurs lack the alpha-blocking effect, and hyper-synchronize the alpha rhythm [30-33]. Alpha hypersynchronization helps explain the augmenting VEP responses during prolonged repeated stimulation (i.e. lack of habituation).

In migraineurs, habituation response normalizes during pain induced by a migraine attack, but it does not normalize during CPT-induced tonic pain. This observation argues against pain being the sole mechanism responsible for ictally normalizing habituation, and suggests that changes in cortical mechanisms regulating the responses to repetitive stimulation differ when they are promoted by a migraine attack or a CPT.

Although we cannot exclude that our findings in migraineurs may derive from reduced activity of intracortical inhibitory circuits, in line with previous neurophysiological studies, we propose as the neural mechanism that abolishes 
the normal VEP habituation and also prevents the block 1 VEP amplitude reduction during tonic pain the patients' low interictal pre-activation levels in the sensory cortices [1]. A low cortical pre-activation level is thought to reflect reduced interictal thalamic and thalamo-cortical activation as disclosed by studies investigating high-frequency oscillations in somatosensory [34] and visual [35] systems. By inducing low-amplitude responses to early stimulation at baseline, the reduced sensory cortex pre-activation level probably prevents a further response decrease during the pain condition. Overall, the disease-related interictal cortical dysfunction in migraine prevents the cortical changes induced by tonic painful stimulation both during pain and after pain ends. Because immediate and longer-lasting cortical changes presumably both reflect CNS plasticity mechanisms that alter synaptic effectiveness in the stimulated cortex through long-term depression or long-term potentiation-like phenomena, our study suggests altered plasticity of sensory cortices in migraine.

Strong support for altered plasticity as a mechanism impairing cortical responsivity in migraine comes also from evidence that the transient cortical dysfunction observed in migraineurs between attacks alters the way the sensory cortices respond to neurophysiological protocols aimed to test cortical plasticity. Depressing visual cortex excitability with low-frequency repetitive transcranial magnetic stimulation increases the phosphene threshold $[36,37]$ and causes a lack of VEP habituation in healthy volunteers [38-40]. In contrast, in patients with migraine, the same rTMS protocols facilitate striate and extrastriate cortical areas resulting in threshold lowering, and also further strengthen the interictal habituation loss. These paradoxical responses call for stringent homeostatic plasticity mechanisms to govern migraineurs' sensory cortex, possibly favouring maladaptive plasticity.

In conclusion, activating the structures subserving tonic pain by CPT leaves the abnormal visual cortex responses to repetitive stimulation in migraineurs unchanged. Because tonic pain modulates visual cortical excitability by activating the monoaminergic brainstem nuclei that in turn modulate activity in thalamic and cortical neurons, we suggest that the lack of pain-induced cortical changes in migraine reflects hypoactivity in monoaminergic brainstem nuclei. By decreasing the level of preactivation in the sensory cortices, this abnormality prevents short-term and longer-term changes in cortical synaptic effectiveness. Having their sensory cortex response patterns locked in a restricted amplitude range possibly means that migraineurs become less able than healthy persons to enact defensive strategies against pain.

Acknowledgment This study was supported by grant from the Franco Michele Puca Award 2007 assigned by the Italian Society for the Study of Headaches (Società Italiana per lo Studio delle Cefalee, SISC).
Conflict of interest None.

\section{References}

1. Schoenen J (1996) Deficient habituation of evoked cortical potentials in migraine: a link between brain biology, behavior and trigeminovascular activation? Biomed Pharmacother 50:71-78

2. Coppola G, Pierelli F, Schoenen J (2009) Habituation and migraine. Neurobiol Learn Mem 92:249-259

3. Thompson RF, Spencer WA (1966) Habituation: a model phenomenon for the study of neuronal substrates of behavior. Psychol Rev 73:16-43

4. Rankin $\mathrm{CH}$, Abrams T, Barry RJ, Bhatnagar S, Clayton D, Colombo J, Coppola G, Geyer MA, Glanzman DL, Marsland S, McSweeney F, Wilson DA, Wu CF, Thompson RF (2009) Habituation revisited: an updated and revised description of the behavioural characteristics of habituation. Neurobiol Learn Mem 92:135-138

5. Aurora SK, Ahmad BK, Welch KMA, Bdardhwaj P, Ramadan NM (1998) Transcranial magnetic stimulation confirms hyperexcitability of occipital cortex in migraine. Neurology 50:11111114

6. Mulleners WM, Chronicle EP, Palmer JE, Koehler PJ, Vredeveld JW (2001) Visual cortex excitability in migraine with and without aura. Headache 41:565-572

7. Kropp P, Gerber WD (1998) Prediction of migraine attacks using a slow cortical potential, the continget negative variation. Neurosci Lett 257:73-76

8. Evers S, Quibeldey F, Grotemeyer KH, Suhr B, Husstedt IW (1999) Dynamic changes of cognitive habituation and serotonin metabolism during the migraine interval. Cephalalgia 19:485-491

9. Siniatchkin M, Kropp P, Gerber WD, Stephani U (2000) Migraine in childhood-are periodically occurring migraine attacks related to dynamic changes of cortical information processing? Neurosci Lett 279:1-4

10. Judit A, Sandor PS, Schoenen J (2000) Habituation of visual and intensity dependence of auditory evoked cortical potentials tends to normalize just before and during the migraine attack. Cephalalgia 20:714-719

11. Kropp P, Gerber WD (1995) Contingent negative variation during migraine attack and interval: evidence for normalization of slow cortical potentials during the attack. Cephalalgia 15:123-128

12. Coppola G, Currà A, Serrao M, Di Lorenzo C, Vatrika M, Parisi V, Pierelli F (2009) Tonic pain abolishes cortical habituation of visual evoked potentials in healthy subjects. J Pain (in press)

13. Petrovic P, Petersson KM, Hansson P, Ingvar M (2004) Brainstem involvement in the initial response to pain. Neuroimage 22:995-1005

14. Watanabe S, Kakigi R, Hoshiyama M, Kitamura Y, Koyama S, Shimojo M (1996) Effects of noxious cooling of the skin on pain perception in man. J Neurol Sci 135:68-73

15. Willer JC, De Broucker T, Le Bars D (1989) Encoding of nociceptive thermal stimuli by diffuse noxious inhibitory controls in humans. J Neurophysiol 62:1028-1038

16. Serrao M, Perrotta A, Bartolo M, Fiermonte G, Pauri F, Rossi P, Parisi L, Pierelli F (2005) Enhanced trigemino-cervical-spinal reflex recovery cycle in pain-free migraineurs. Headache 45:1061-1068

17. Sandrini G, Rossi P, Milanov I, Serrao M, Cecchini AP, Nappi G (2006) Abnormal modulatory influence of diffuse noxious inhibitory controls in migraine and chronic tension-type headache patients. Cephalalgia 26:782-789 
18. de Tommaso M, Sardaro M, Pecoraro C, Di Fruscolo O, Serpino C, Lamberti P, Livrea P (2007) Effects of the remote C fibres stimulation induced by capsaicin on the blink reflex in chronic migraine. Cephalalgia 27:881-890

19. Panconesi A (2008) Serotonin and migraine: a reconsideration of the central theory. J Headache Pain 9:267-276

20. Tassorelli C, Sandrini G, Cecchini AP, Nappi RE, Sances G, Martignoni E (2002) Changes in nociceptive flexion reflex threshold across the menstrual cycle in healthy women. Psychosom Med 64:621-626

21. Sandrini G, Milanov I, Malaguti S, Nigrelli MP, Moglia A, Nappi G (2000) Effects of hypnosis on diffuse noxious inhibitory controls. Physiol Behav 69:295-300

22. Backonja M, Howland EW, Wang J, Smith J, Salinsky M, Cleeland CS (1991) Tonic changes in alpha power during immersion of the hand in cold water. Electroencephalogr Clin Neurophysiol 79:192-203

23. Chang PF, Arendt-Nielsen L, Chen AC (2002) Dynamic changes and spatial correlation of EEG activities during cold pressor test in man. Brain Res Bull 57:667-675

24. Chen CAN, Rappelsberger P (1998) Topology of EEG coherence changes may reflect differential neural network activation in cold and pain perception. Brain Topogr 11:125-132

25. Ferracuti S, Seri S, Mattia D, Cruccu G (1994) Quantitative EEG modifications during the cold water pressor test: hemispheric and hand differences. Int J Psychophysiol 17:261-268

26. Groves PM, Thompson RF (1970) Habituation: a dual-process theory. Psychol Rev 77:419-450

27. Mesulam MM (1990) Large-scale neurocognitive networks and distributed processing for attention, language and memory. Ann Neurol 28:597-613

28. Peyron R, Garcia-Larrea L, Gregoire MC, Costes N, Convers P, Lavenne F, Mauguiere F, Michel D, Laurent B (1999) Haemodynamic brain responses to acute pain in humans: Sensory and attentional networks. Brain 122:1765-1780

29. Sappey-Marinier D, Calabrese G, Fein G, Hugg JW, Biggins C, Weiner MW (1992) Effect of photic stimulation on human visual cortex lactate and phosphates sing $1 \mathrm{H}$ and 51P magnetic resonance spectroscopy. J Cereb Blood Flow Met 12:584-592

30. Simon RH, Zimmerman AW, Tasman A, Hale MS (1982) Spectral analysis of photic stimulation in migraine. Electroencephalogr Clin Neurophysiol 53:270-276

31. Tsounis S, Varfis G (1992) Alpha rhythm power and the effect of photic stimulation in migraine with brain mapping. Clin Electroencephalogr 23:1-6
32. de Tommaso M, Sciruicchio V, Guido M, Sasanelli G, Puca F (1999) Steady state visual-evoked potentials in headache: diagnostic value in migraine and tension-type headache. Cephalalgia 19:23-26

33. de Tommaso M, Marinazzo D, Nitti L, Pellicoro M, Guido M, Serpino C, Stramaglia S (2007) Effects of levetiracetam vs topiramate and placebo on visually evoked phase synchronization changes of alpha rhythm in migraine. Clin Neurophysiol 118:2297-2304

34. Coppola G, Vandenheede M, Di Clemente L, Ambrosini A, Fumal A, De Pasqua V, Schoenen J (2005) Somatosensory evoked highfrequency oscillations reflecting thalamo-cortical activity are decreased in migraine patients between attacks. Brain 128:98-103

35. Coppola G, Ambrosini A, Di Clemente L, Magis D, Fumal A, Gérard P, Pierelli F, Schoenen J (2007) Interictal abnormalities of gamma band activity in visual evoked responses in migraine: an indication of thalamocortical dysrhythmia? Cephalalgia 27:13231330

36. Brighina F, Piazza A, Daniele O, Fierro B (2002) Modulation of visual cortical excitability in migraine with aura: effects of $1 \mathrm{~Hz}$ repetitive transcranial magnetic stimulation. Exp Brain Res 145:177-181

37. Fierro B, Ricci R, Piazza A, Scalia S, Giglia G, Vitello G, Brighina F (2003) $1 \mathrm{~Hz}$ rTMS enhances extrastriate cortex activity in migraine: evidence of a reduced inhibition? Neurology 61:1446-1448

38. Bohotin V, Fumal A, Vandenheede M, Gérard P, Bohotin C, Maertens de Noordhout A, Schoenen J (2002) Effects of repetitive transcranial magnetic stimulation on visual evoked potentials in migraine. Brain 125:912-922

39. Fumal A, Bohotin V, Vandenheede M, Seidel L, de Pasqua V, de Noordhout AM, Schoenen J (2003) Effects of repetitive transcranial magnetic stimulation on visual evoked potentials: new insights in healthy subjects. Exp Brain Res 150:332-340

40. Fumal A, Coppola G, Bohotin V, Gérardy PY, Seidel L, Donneau AF, Vandenheede M, Maertens de Noordhout A, Schoenen J (2006) Induction of long-lasting changes of visual cortex excitability by five daily sessions of repetitive transcranial magnetic stimulation (rTMS) in healthy volunteers and migraine patients. Cephalalgia 26:143-149 